\title{
Incidentaloma: The Case of an Asymptomatic Mediastinal Ganglioma
}

Burton Jared Tabaac ${ }^{1^{*}}$, Matthew Jaffa ${ }^{2}$, Christopher Bosse ${ }^{3}$ and William Ward ${ }^{3}$

${ }^{1}$ Rutgers Robert Wood Johnson Medical School, New Brunswick, NJ, USA

${ }^{2}$ University of Maryland Medical Center, Department of Neurology, Baltimore, MD 21201, USA

${ }^{3}$ Abington Jefferson Health, Department of Pulmonary and Critical Care, Abington, PA 19001, USA

*Corresponding author: Burton Jared Tabaac, 373 E Main Street, Suite 314, Somerville, NJ 08876, USA, Tel: 1-267-334-4040; Fax: 888-450-7730; E-mail: Burton.tabaac@gmail.com

Received date: Dec 30, 2015; Accepted date: Jan 04, 2016; Published date: Jan 13, 2016

Copyright: (02016 Tabaac BJ, et al. This is an open-access article distributed under the terms of the Creative Commons Attribution License, which permits unrestricted use, distribution, and reproduction in any medium, provided the original author and source are credited.

\begin{abstract}
Mediastinal masses of neuronal origin are exceedingly rare. We present the case of a 72-year-old woman, who was seen and evaluated by a pulmonologist, complaining of shortness of breath. The patient was found to have a mass visualized by computed tomography which was biopsied for pathologic identification. Upon microscopic examination of the transbronchial biopsy, neural tissue was observed. It is likely that the source of this ganglioma was of phrenic nerve origin.
\end{abstract}

Keywords: Schwann cell; Pathology; Histopathology

\section{Introduction}

Incidental tumors found in the mediastinum of neurogenic origin are relatively rare; though they do account for $10-34 \%$ of mediastinal masses in general [1]. A review of the surgical literature details the multifaceted approaches to their removal and reveals that tumors originating from the vagus and phrenic nerves are among the more rare [2,3]. Schwannomas are benign, lobulated, and spherical neoplasms composed of Schwann cells that originate from the neural sheath, ganglionic, or paraganglionic cells [2]. Typically, schwannomas only begin to cause perceptible symptoms when they compress surrounding structures [4]. We present the case of a woman initially complaining of dyspnea and a decreased DLCO on pulmonary function testing requiring CT imaging of the chest. Upon imaging, an indeterminate mass was observed in the mediastium that required biopsy, and status post biopsy, revealed nerve tissue.

\section{Case Report}

A 72-year-old woman with past medical history significant for obstructive sleep apnea (OSA) initially diagnosed in 2010 and a repeat study in 2014 revealing no evidence of OSA, hypertension, hyperlipidemia and a five pack-year history of smoking was evaluated for progressive shortness of breath by a pulmonary specialist at the request of her primary care physician. At the time of her visit, the patient's dyspnea had made ascending one flight of stairs and occasional activities of daily living difficult. She had been treated with three different medications for asthma over a 10-year period and reported occasional chest pain but never dyspnea at rest or orthopnea. Advair and Dulera were intolerable secondary to adverse effects involving the patient's eyes. Treatment with Albuterol and Spiriva had not been attempted. A previous CVA left the patient with residual dysarthria and right hand weakness. The patient denied dysphagia or aspiration events.

On examination, the patient was found to be alert and oriented without respiratory distress. The pupils were equal and reactive to light with intact extraocular muscles. Her nasal passages were boggy, but without drainage, and there were no significant abnormalities of the oropharynx. Auscultation of the left carotid artery revealed a bruit. No abnormal lymph nodes were appreciated in the neck, supraclavicular or axillary regions. The patient's lungs were clear to auscultation without rales or wheezes. Heart examination revealed regular rhythm without murmur or rub. Both abdominal and gross neurological exams were benign.

Pulmonary function testing demonstrated a borderline obstructive defect of the small airways with improvement after bronchodilator use. Lung volumes were found to be within normal limits, and DLCO was severely reduced consistent with a gas exchange abnormality or component of emphysema. A six-minute walk test proved that the patient was able to walk 936 feet on room air with a noted oxygen desaturation from $99 \%$ at rest to a minimum of $93 \%$ with a maximum heart rate of 89 beats per minute. The patient stopped to rest for 30 seconds during the test due to foot pain attributable to tendinitis.

Imaging with computed tomography of the thorax revealed no evidence for an acute pulmonary embolus, however, there was a $9 \mathrm{~mm}$ left lung nodule and a $5 \mathrm{~mm}$ right lung nodule observed. Additionally, an indeterminate $1.2 \times 1.8 \mathrm{~cm}$ soft tissue density was seen abutting the right pericardium, adjacent to a right pulmonary vein, as it extended into the left atrium (Figure 1). An electronavigational bronchoscopy with transbronchial biopsy via needle brush, and bronchialveolar lavage of the right lower lobe, followed by endobronchial ultrasound (EBUS) evaluation of the right lower lobe nodule with additional EBUS guided needle biopsies and bronchoscopy inspection was performed. Pathology of the samples revealed tiny fragments of benign bronchial tissue and fragments of benign nerve or nerve ganglia tissue (Figure 2). 


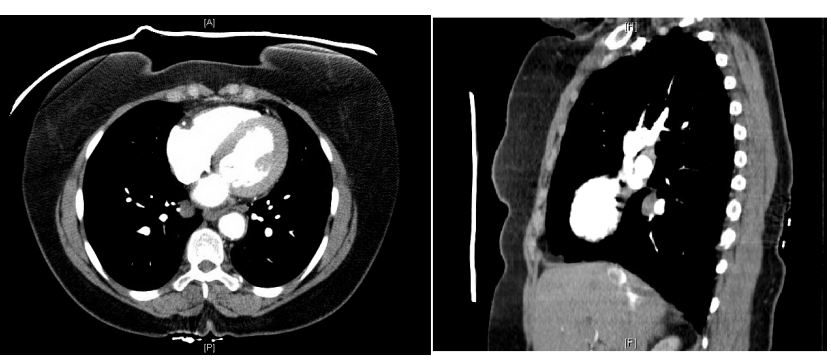

Figure 1: Computed tomography of thorax (L: axial, R: sagittal).

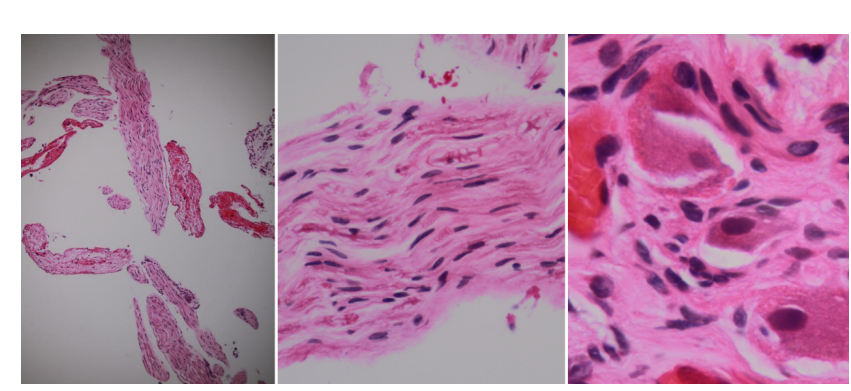

Figure 2: H\&E stained slide showing neural biopsy; from L to R: 100x, 400x, 1000x (cluster of ganglion cells, under oil).

\section{Discussion}

Masses found on routine imaging of the thorax are determined to be of neurogenic origin approximately $20 \%$ of the time [5]. Though routinely benign, the masses are frequently resected. Our patient's mass was biopsied via transbronchial biopsy and reveals what we believe to be a Schwannoma of the right phrenic nerve. Schwannomas are typically found in the posterior mediastinum, and in reviewing the recent literature, we conclude that it is a relative rarity to find schwannomas in the middle mediastinum. Only several series of case reports have shown the site of origination of mediastinal tumors to be the phrenic nerve [6]. As in most cases of mediastinal gangliomas, the lesion biopsied in this patient was found to be benign and asymptomatic likely secondary to its relatively small size. Gangliomas of the mediastinum typically remain asymptomatic until their size begins to encroach other structures. In other reported cases, a variety of benign symptoms were noted including cough, hoarseness or eventration of the diaphragm. One interesting case described a patient with a twenty year history of intractable hiccups that were cured after the resection of a tumor from the patient's phrenic nerve [7].

Pathologic examination of our patient's transbronchial biopsy tissue shows the presence of small detached fragments of normal-appearing nerve and nerve ganglion cells. Scant benign bronchial tissue should not contain nerve fragments, thus representing an abnormal finding. Given the location of this biopsy specimen, it is highly likely that this ganglioma developed from the phrenic nerve and less likely to be from the sympathetic chain ganglion or other neural origin.

Surgery is indicated for the removal of such tumors only when they are found to be symptomatic or present with the rare findings of malignancy. In our patient's case, she will be followed with a repeat CT scan of the chest 6 months following her initial biopsy to asses for change in the size, calcification, or encroachment of the lesion. Imaging will also be necessary to follow and observe any alterations of the pulmonary nodule in the left lung that was not biopsied during this visit. Video-assisted thoracic surgery is a safe technique for benign mediastinal tumors, typically those in the middle and posterior mediastinum [8].

\section{References}

1. Sasaki K, Kohno T, Mun M, Yoshiya T (2008) Thoracoscopic Removal of Middle Mediastinal Schwannoma Originating from Recurrent Nerve. Thorac Cardiov Surg 56: 375 - 377 .

2. Rammos K, Rammos S, Foroulis C, Zaramboukas T (2009) Schwannoma of the vagus neve, a rare middle mediastinal neurogenic tumor: case report. Journal of Cardiothoracic Surgery 4: 68.

3. Muinuddeen K, Baltzer JW, Zama N (2001) Diaphragmatic eventration: an uncommon presentation of a phrenic nevre schwannoma. Chest 119: $1615-1616$.

4. Ortigara L, Rosemberg N, Siqueira R, Neto F (2006) Resection of a mediastinal schwannoma using videoaisted thoracoscopy. J bras pneumol 32: $172-175$.

5. Orki A, Ozdemir A, Ersev AA, Kutlu CA (2012) A Schwannoma in the Middle Mediastinum Originated from the Phrenic Nerve. Indian J Surg 74: $199-200$.

6. Gurkok S, Genc O, Gozubuyuk A, Caylak H, Kavakli K (2009) Schwannoma arising from the right phrenic nerve. Turkish Jounal of Thoracic and Cardiovascular Surgery 17: 49-50.

7. Gilani SM, Danforth RD (2012) Intractable hiccups: A rare presentation of phrenic nerve schwannoma. European Annals of Otorhinolaryngology, Head and Neck Diseases 129: 331-333.

8. Demmy TL, Krasna MJ, Detterbeck FC, Kline GG, Kohman LJ, et al. (1998) Multicenter VATS experience with mediastinal tumors. Ann Thorac Surg 66: 187-192. 